\title{
Synthesis and Characterization of Polymeric Composites Embeded with Silver Nanoparticles
}

\author{
Hemant K. Chitte ${ }^{1}$, Narendra V. Bhat ${ }^{2 *}$, Narayan S. Karmakar ${ }^{3}$, \\ Dushyant C. Kothari ${ }^{3}$, Ganesh N. Shinde ${ }^{4}$ \\ ${ }^{1}$ Dnyanasadhana College, Thane, India \\ ${ }^{2}$ Bombay Textile Research Association, Mumbai, India \\ ${ }^{3}$ Centre for Nanosciences and Nanotechnology, University of Mumbai, Mumbai, India \\ ${ }^{4}$ Indira Gandhi College, New Nanded, India \\ Email: *nvkbhat@gmail.com
}

Received November 30, 2011; revised December 12, 2011; accepted January 2, 2012

\begin{abstract}
Silver nanoparticles were synthesized by chemical reduction method. The Ag nanoparticles (AgNP) were characterized using UV-Vis spectroscopy which shows an absorption band at $420 \mathrm{~nm}$ confirming the formation of nanoparticles. For any practical application of the silver nanoparticles it is necessary to stabilize it which can be done by making a composite. In the present studies three polymers were chosen such that AgNP could be put to some practical use. Polyvinyl Alcohol (PVA), Polypyrrole (Ppy) and Carboxymethyl cellulose (CMC) are important for use in textiles, electronics and food/drug technologies respectively. Polymeric composites of PVA, PPy, and CMC were prepared by mixing the aqueous solutions of the respective polymers and the colloidal suspension of pre-formed silver nanoparticles. Various compositions containing $1 \%$ to $5 \%$ of Ag nanoparticles were prepared. Thin films of these composites were characterized by UV-Vis spectroscopy, X-ray diffraction and Scanning electron microscopy. X-ray diffraction showed the presence of the peaks at $2 \theta$ values of $38.1^{\circ}, 44.2^{\circ}, 64.4^{\circ}$ and $78.2^{\circ}$ corresponding to cubic phase of silver metal. SEM photographs revealed the presence of Ag nanoparticles of sizes varying from 40 to $80 \mathrm{~nm}$. The electrical conductivity of these materials was studied using the four probe method. The conductivity was found to increase from $10^{-6}$ for control samples to $10^{-3} \mathrm{~S} / \mathrm{cm}$ after the formation of the nanocomposites.
\end{abstract}

Keywords: Silver Nanoparticles; Composites; Polypyrrole; PVA; CMC; Microscopy

\section{Introduction}

Nanotechnology has assumed a great importance in recent years on account of its possible applications in several areas such as electronics, pharmacy, computers, catalysis, biotechnology etc. [1]. Metallic nanoparticles of Ag, Au, $\mathrm{Cu}$ and $\mathrm{Zn}$ have been the focus of specific interest due to its unique properties such as antibacterial, optical bi-stability, photoresponsivity etc. In spite of such importance the instability of nanoparticles gives rise to aggregation which deters its use for specific applications.

Various methods for stabilizing and capping of nanoparticles have been reported [2]. Coating of nanoparticle and its surface modification with functional polymers have been achieved to give resistance to oxidation and possibility of embedding such modified nanoparticles in different composites. The interest in nano-coating is on account of combination of the properties of two or more materials involved with the emphasis on the fact that one of the materials (shell) will determine the surface proper-

*Corresponding author. ties of the particle while the other i.e. the core is completely encapsulated by the shell. Although the core will not contribute to surface property it will be responsible for optical, electrical and other properties of the composites. It is also important to take into account the possible interaction between the core and shell, so that the unique combination can be understood. Another main benefit of coating nanoparticle is avoidance of spread of nanoparticle to the environment due to bonding between the substrate and coated nanoparticle. Thus the nanoparticle may be safer to move to different places and use for specific purposes.

For any practical application of the silver nanoparticles it is necessary to stabilize the NPs which can be done by making a composite. In the present studies three polymers were chosen such that AgNP could be put to some practical use. PVA, Ppy and CMC are important for use in textiles, electronics and food/drug technologies respectively. In the present paper we have synthesized the composites of silver nanoparticles with Polyvinyl alcohol, Polypyrrole and Carboxymethyl cellulose. The procedure 
adopted was to coat the pre-formed Ag nanoparticles with the polymers in suspension. The nanocomposites thus formed were characterized using UV-Vis spectroscopy (UV-Vis), X-ray diffraction (XRD) and Scanning electron Microscopy (SEM). The advantages of these nanocomposites over the control polymer and industrial applications will be reported in a separate communication.

\section{Experimental}

\subsection{Preparation of Silver Nanoparticles}

$\mathrm{AgNO}_{3}$ was dissolved in double distilled water to give a solution of $0.001 \mathrm{M}$ solution. Trisodium citrate $(0.02 \mathrm{M})$ was used as reducing agent. To $100 \mathrm{ml}$ of silver nitrate solution was added to trisodium citrate solution drop by drop, over a period of $30 \mathrm{~min}$ and maintained at $80^{\circ} \mathrm{C}$. The stirring was continued for $1 \mathrm{hr}$. It was found that solution turns yellow. The yellow colour confirms the formation of silver nanoparticles [3]. A large quantity of such solution was made and served as the stock solution for other experiments.

\subsection{Coating of Ag Nanoparticles by Polymers}

The coating of silver nanoparticle by polypyrrole was performed by addition of $4 \mathrm{ml}$ of pyrrole to $600 \mathrm{ml}$ of silver nanoparticle solution. The solution was stirred continuously during and after addition and it was observed that the conversion of pyrrole to polypyrrole takes place within $15 \mathrm{~min}$. However the stirring was continued for another $1 \mathrm{hr}$ so that the pyrrole fully polymerizes and coats the Ag nanoparticle evenly. The color of the solution changes from yellow to steel grey to black. The obtained solution was left in the ambient condition for another $24 \mathrm{hr}$ before the precipitated material was filtered out and washed.

Polymeric composites of Polyvinyl alcohol and Carboxymethyl cellulose were prepared by mixing the aqueous solutions of the respective polymers and the colloidal suspension of pre-formed silver nanoparticles. The solutions of PVA and CMC were made in water using $4 \%$ of powders $(\mathrm{w} / \mathrm{w})$ and stirring for one hour at $80^{\circ} \mathrm{C}$. To these solutions were added the solution of AgNP to get various compositions containing $1 \%$ to $5 \%$ of $\mathrm{Ag}$. Thin films of these solutions were casted on glass plates for further analysis.

The results described in this paper are for compositions all containing $1 \% \mathrm{w} / \mathrm{w}$ of $\mathrm{Ag}$ nanoparticles.

\subsection{Methods of Characterization}

All the preparations i.e. pure silver nanoparticles and the composites were characterized by UV-Vis, XRD, SEM and TEM. For this purpose UV-Vis Spectrometer of Var- ian make Cary 5000 which could scan from $175 \mathrm{~nm}$ to $3300 \mathrm{~nm}$ was used. Diluted solution of nanoparticles was filled into the quartz cuvette to obtain the spectrum. PAN analytical Xpert pro X-ray diffractometer with $\mathrm{Cu}$ tube was used for recording the XRD pattern. The specimen in the form of thin coating on glass plate or PET film was prepared and used for scanning in the range of $10^{\circ}$ to $80^{\circ}$. Scanning electron micrographs were obtained using JEOL SEM model JSM 5400. The samples were mounted on the stub and coated with a thin film of gold before observations. Transmission electron micrographs were obtained by using Philips TEM CM200 operating at voltage of $200 \mathrm{kV}$. Structures of different polymers used for coating of silver nanoparticles are shown in Figure 1.

\section{Results and Discussions}

\subsection{Composite of Silver Nanoparticles with Polypyrrole}

\subsubsection{UV-Vis Spectroscopy}

The dispersions of silver nanoparticles display intense colors due to the plasmon resonance absorption. The surface of a metal is like plasma, having free electrons in the conduction band and positively charged nuclei. Surface plasmon resonance is a collective excitation of the electrons in the conduction band near the surface of the nanoparticles. Electrons are limited to specific vibrations modes by the particle's size and shape. Therefore, metallic nanoparticles have characteristic optical absorption spectra in the UV-Vis region [3].

The UV-Vis absorption spectrum of pure silver nanoparticle is shown in Figure 2. One can clearly see strong absorption at $417 \mathrm{~nm}$, confirming the formation of silver nanoparticles. The peak at $417 \mathrm{~nm}$ is attributed to the surface plasmon resonance.

UV-Vis Spectra for the solution in which pyrrole is added is shown in Figure 3. Curve A of Figure 3 is UVVis spectrum of silver nanoparticle solution. It shows the strong absorption peak at $417 \mathrm{~nm}$. Curve B of Figure 3 corresponds to Ag nanoparticle solution in which pyrrole

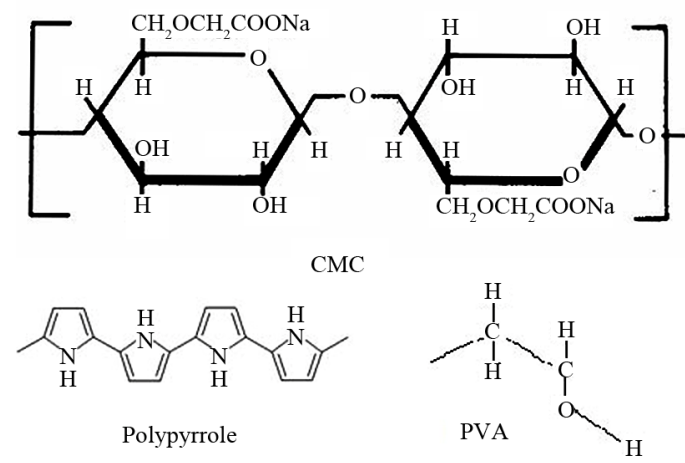

Figure 1. Molecular structures of Carboxy methyl cellulose, Polypyrrole and Polyvinyl alcohol. 


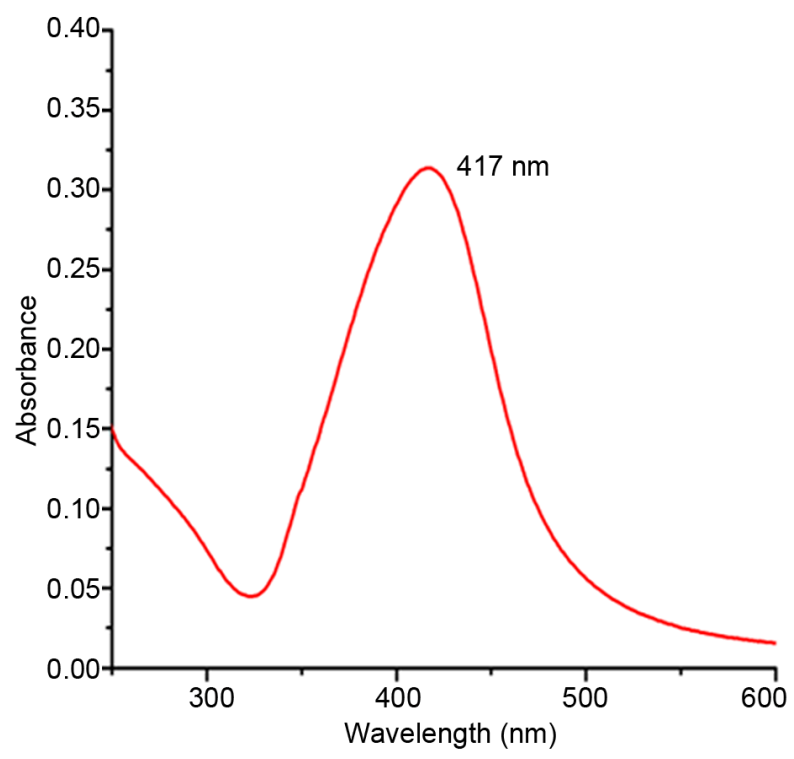

Figure 2. UV-Vis absorption spectra of colloid solution of Ag nanoparticles.

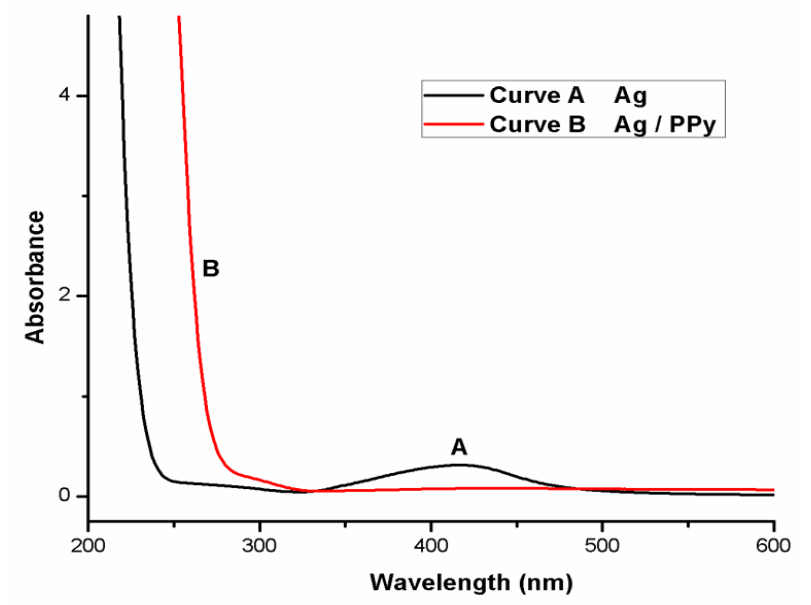

Figure 3. A: UV-Vis absorption spectra of colloid solution of Ag nanoparticles; B: Ag/PPy nanoparticle.

was added and allowed to polymerize for sufficient duration. It can be noted that the peak at $417 \mathrm{~nm}$ observed earlier for pure Ag nanoparticles has now disappeared. The color of the solution changes from yellow to steel grey to black. This is due to fact that the PPy coats around Ag nanoparticles and as the color of polypyrrole is black, the entire solution and the precipitated composite gets black color. In addition the strong scattering from the PPy shell possibly screened the surface and as a result the plasmon resonance does not occur.

\subsubsection{X-Ray Diffraction Analysis}

$\mathrm{X}$-ray diffraction pattern for pure silver nanoparticle reported in literature shows four distinct diffraction peaks at $38.7^{\circ}, 44.1^{\circ}, 64.6^{\circ}$ and $78.3^{\circ}$ [JCPDS No 03-0931].
These correspond to cubic, crystalline structure of silver and are assigned to (111), (200), (220) and (311) planes of silver respectively. Figure 4 shows X-ray diffraction pattern of PPy coated Ag nanoparticle. This shows all the four characteristic peaks due to silver crystalline structure and in addition a broad peak in the region $20^{\circ}$ to $24^{\circ}$ due to the amorphous structure of polypyrrole [4].

The four peaks observed for $\mathrm{Ag} / \mathrm{PPy}$ are at $2 \theta$ angles of $37.8^{\circ}, 44.0^{\circ}, 64.2^{\circ}$ and $77.1^{\circ}$. These values are slightly lower than those reported in JCPDS. The particle size of the silver nanoparticle was calculated from the line broadening using the Scherrer's formula $\mathrm{D}=\mathrm{k} \lambda / \beta \cos \theta$. The sizes were calculated from all the four peaks and were found to be $27 \mathrm{~nm}, 23 \mathrm{~nm}, 23 \mathrm{~nm}$ and $20 \mathrm{~nm}$ respectively. The XRD pattern did not change even after 60 days after preparation. This indicated that the PPy coating prevented the Ag nanoparticles from being oxidized even in air, compared with the uncoated Ag nanoparticles, which is evidence of the protection that the PPy exerts against oxidation of the Ag core. Thus, quite stable Ag nanoparticles could be fabricated with PPy coating.

\subsubsection{Transmission Electron Microscopy}

Figure 5(a) shows TEM image of pure silver nanoparticle and one can see minimum size of Ag nanoparticle as about $20 \mathrm{~nm}$. Large particles of different sizes in the range from $20 \mathrm{~nm}$ to $50 \mathrm{~nm}$ were also seen, which are probably due to aggregation or clustering of the nanoparticle. Figure 5(b) shows the TEM image of polypyrrole coated silver nanoparticle. One can note beautiful core/ shell structure. The Ag nanoparticles seem to be embedded in the PPy matrix. This feature is very much different from pure polypyrrole, whose morphology is mainly spherulitic or globular with the average size of $0.8 \mathrm{mi}-$ crons, as seen in Figure 5(c).

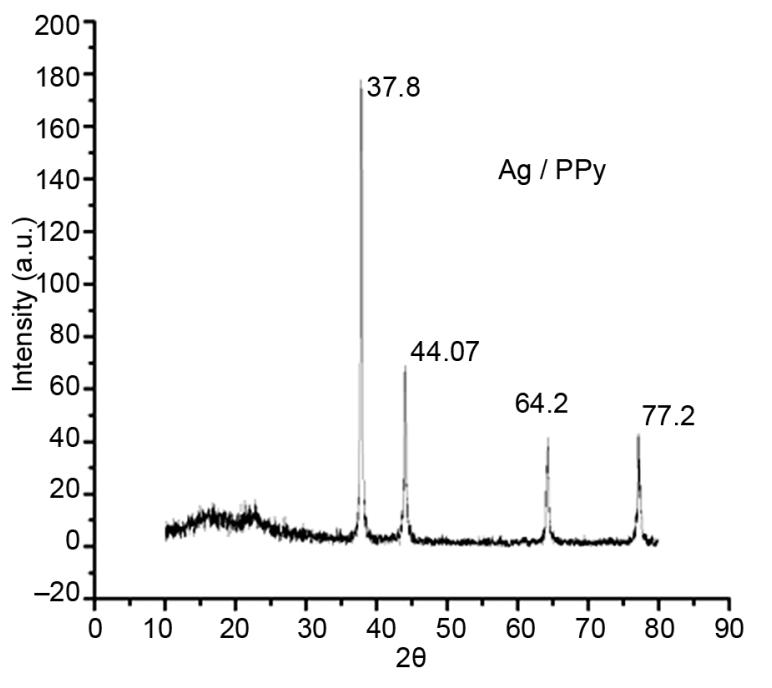

Figure 4. XRD Patterns of Ag/Ppy nanoparticles. 


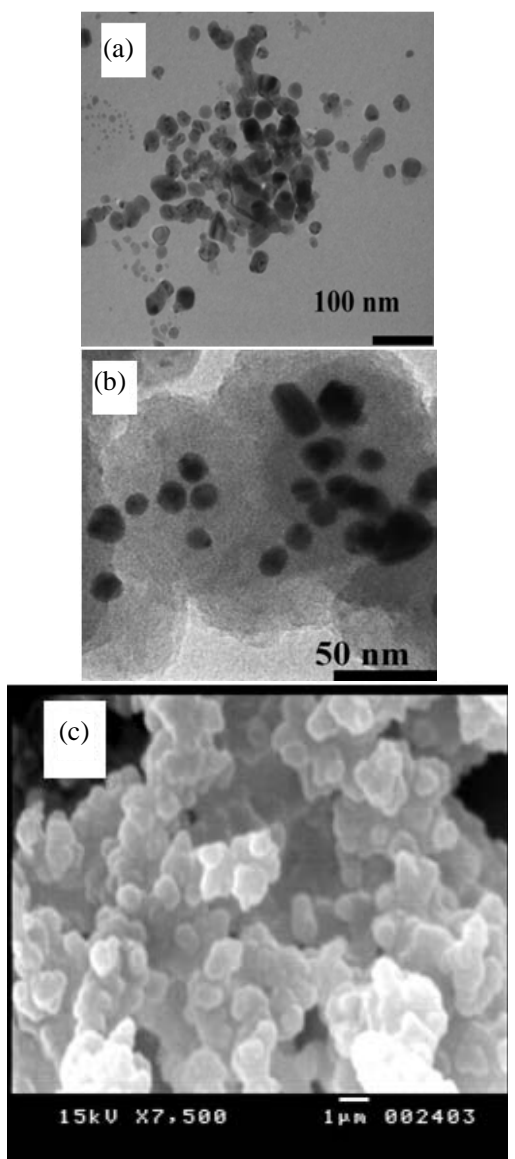

Figure 5. Micrographs (a) Pure Ag nanoparticles, (b) Ag nanoparticles coated with PPy and (c) SEM of pure Ppy.

\subsection{Composites of Silver Nanoparticles with PVA}

\subsubsection{UV-Vis Spectroscopy of Ag NP/ PVA}

The nanocomposite films of various compositions were checked for their absorption patterns. The composite films exhibit a broad surface Plasmon absorption band peaking at approximately $420 \mathrm{~nm}$ (Figure 6). This result is in agreement with the optical absorption spectra of $\mathrm{Ag}$ nanoparticles embedded in different polymer matrices like polyacrylonitrile, nylon etc. The shift to the longer wavelength and broadening of the band upon incorporation of Ag nanoparticles into PVA can be induced by agglomeration of the Ag nanoparticles or change of the dielectric properties of the surrounding environment.

\subsubsection{X-Ray Diffraction}

$\mathrm{X}$-ray diffraction pattern of the nanocomposite films showed many peaks at different $2 \theta$ values as shown in Figure 7. The peaks around $20^{\circ}$ have resulted from the crystalline parts of PVA molecules. (In fact it is a group of three peaks corresponding $19^{\circ}, 21^{\circ}, 23^{\circ}$, not seen resolved here due to reduced size of the figure). This agrees well with the previous reported work for the PVA [5]. In addition very intense peaks were observed at $38^{\circ}, 44^{\circ}, 64^{\circ}$ and $78^{\circ}$ which are due to cubic form of silver and also agrees well with the literature [JCPDS No 030931]. Thus we can see that a homogeneous nanocomposite film of PVA with Ag NP has been formed.

The nanocomposite of PVA/Ag was observed using AFM. Figures 8(a) and (b) show the surface morphology of the films in 2 and 3 dimensions. One can see easily the silver nanoparticles of size $40 \mathrm{~nm}$ protruding out of the surface. This structure is certainly much different from that for pure PVA, which shows mainly a planar surface as seen in Figure 8(c).

\subsection{Composites of Silver Nanoparticles with CMC}

The nanocomposites of CMC + Ag were also investigated

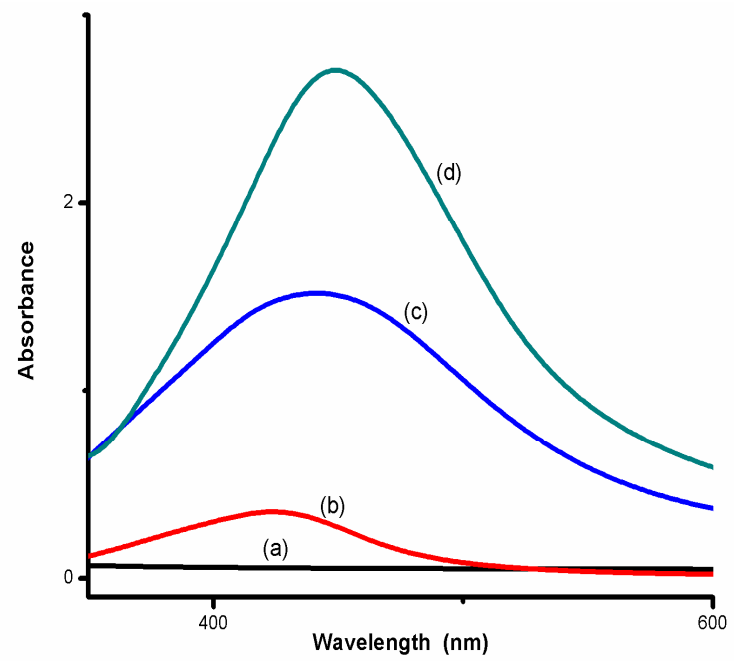

Figure 6. UV/Vis spectra of PVA/Ag nanocomposites of different concentrations (a) Control PVA; (b) $0.2 \%$ Ag content; (c) $0.5 \%$ Ag content (d) $1.0 \%$ Ag content.

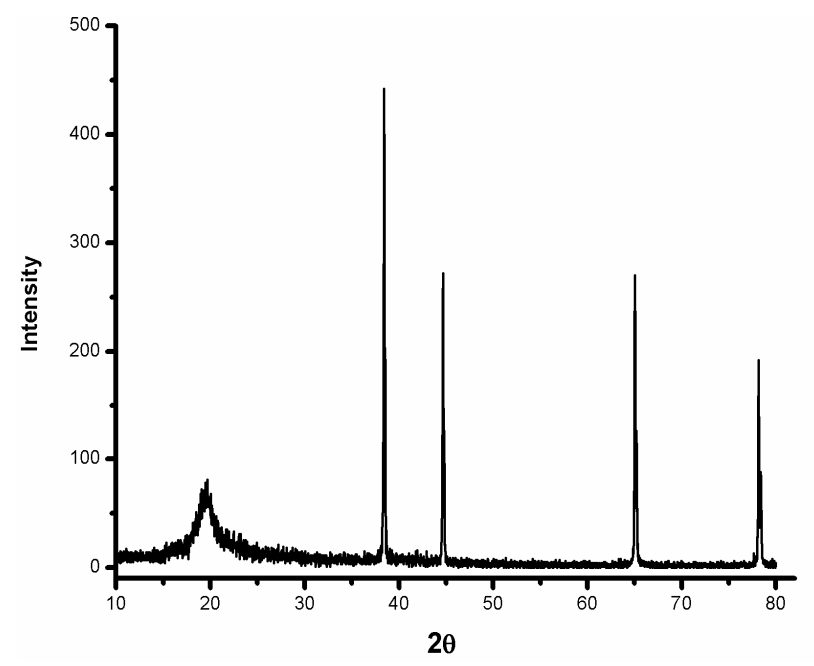

Figure 7. X-ray diffractogram of PVA/ Ag nanocomposites. 


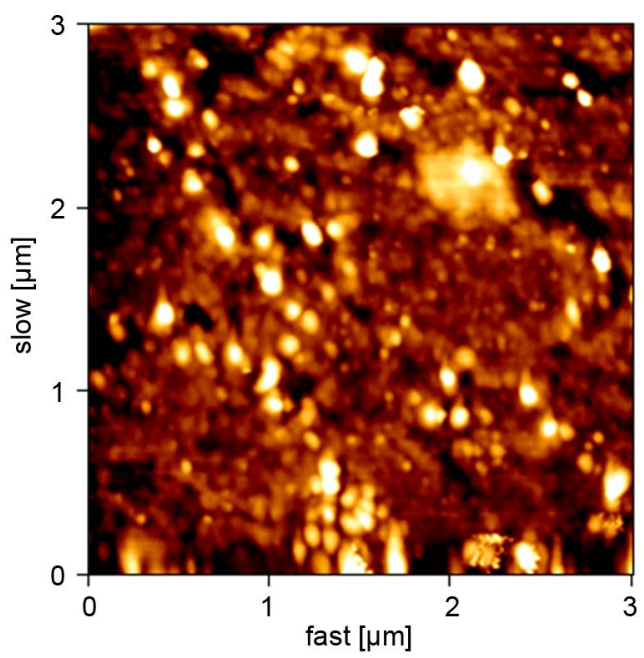

(a)

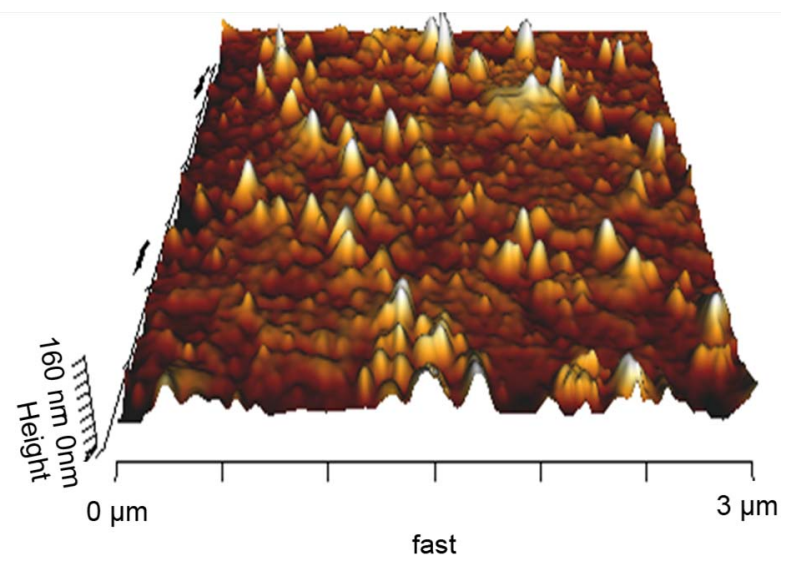

(b)

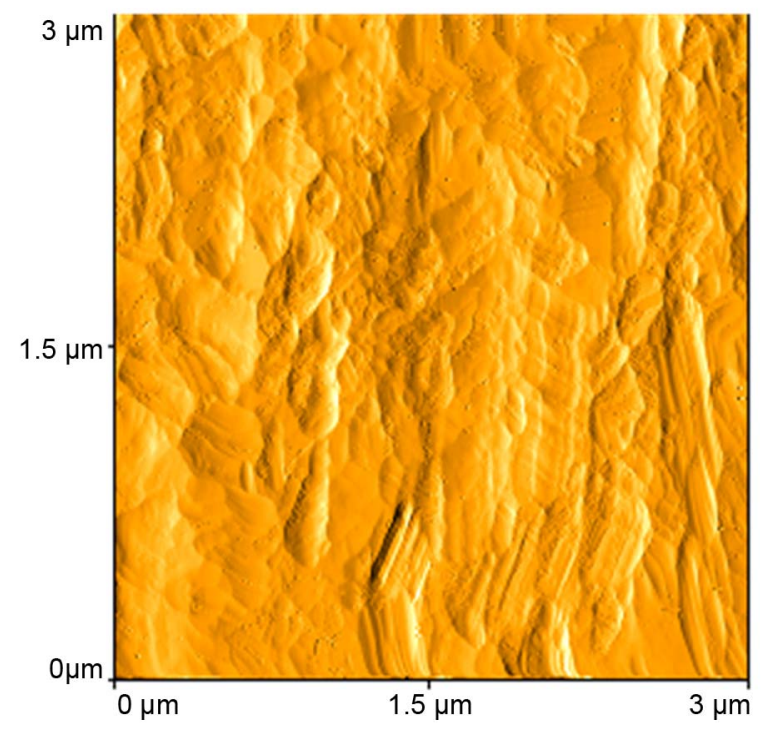

(c)

Figure 8. Morphological features of composite of AgNP+ PVA seen using AFM: (a) Planar surface; (b) 3-D view of surface and (c) of pure PVA film.
$\mathrm{CMC}+\mathrm{Ag}$ Nano 20

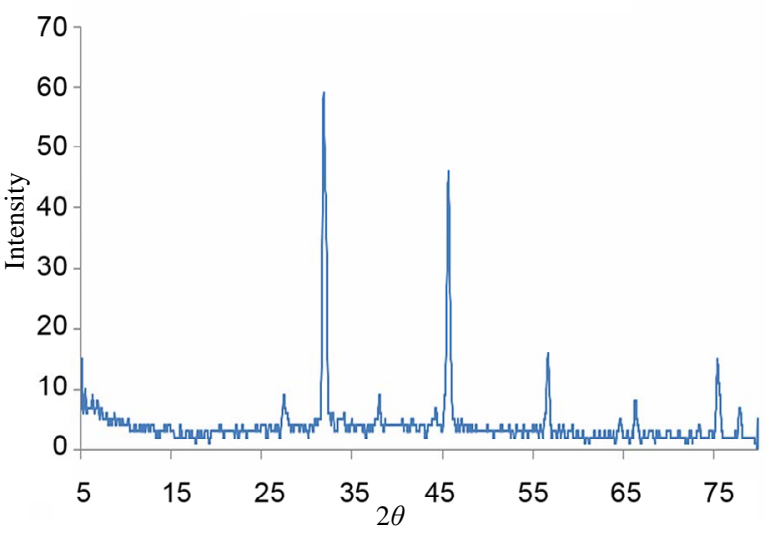

Figure 9. X-ray diffraction pattern of CMC + Ag nanoparticles.

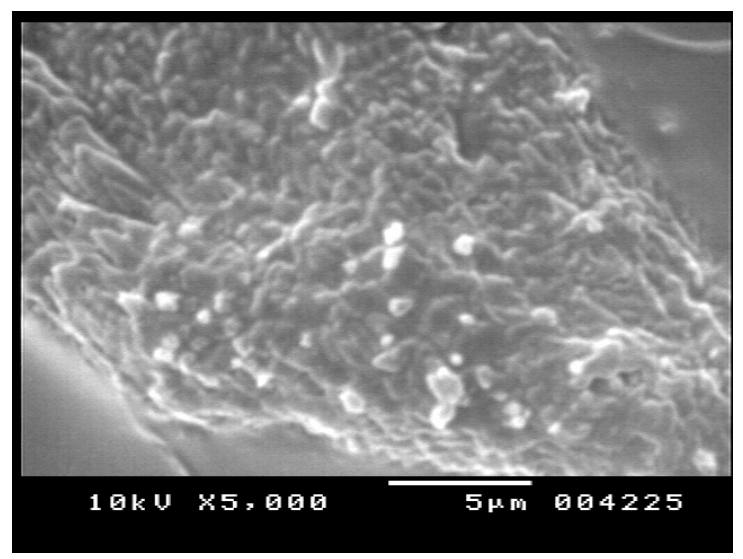

(a)

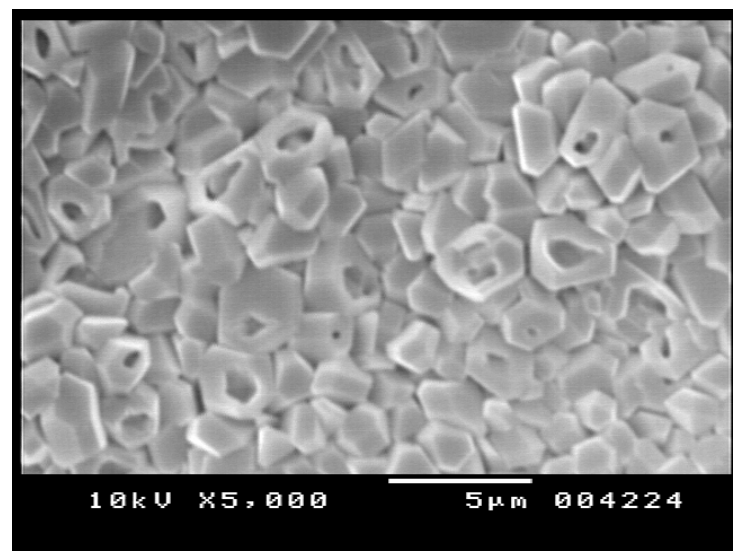

(b)

Figure 10. SEM micrographs of (a) Pure CMC and (b) CMC + Ag nanoparticles conclusions.

using the UV/Vis spectroscopy and one could easily observe the absorption band at $420 \mathrm{~nm}$. The X-ray diffracttion also revealed that a good composite has been formed as seen by the presence of peaks due to silver (Figure 9).

Morphological studies using SEM provided interesting 
information about the granular structure of pure CMC changing to square type of formations with silver nanoparticles embedded in the center (Figure 10).

Polymeric Nanocomposites containing Ag NP could be prepared with three industrially important polymers , namely, Polyvinyl alcohol, polypyrrole and Carboxymethyl cellulose. The Ag NP gets coated with the polymers which improves its stability and functionality. Significant changes were noted in the surface morphology and homogeneous distribution of Ag NP was observed.

\section{Acknowledgements}

The authors wish to express thanks to Director, BTRA for his interest and necessary permission. Thanks are also due to Mr. A. V. Gore and Mr. V. E. Walunj for the help during experimental work.

\section{REFERENCES}

[1] A. Franks, "Review Article: Nanotechnology,” Journal of
Physics E: Scientific Instruments, Vol. 20, No. 12, 1987, pp. 1442-1451. doi:10.1088/0022-3735/20/12/001

[2] E. Filipo, A. Serra and D. Manno, "Poly(Vinyl Alcohol) Capped Silver Nanoparticles as Localized Surface Plasmon Resonance-Based Hydrogen Peroxide Sensor," Sensors and Actuators B: Chemical, Vol. 138, No. 2, 2009, pp. 625-630. doi:10.1016/j.snb.2009.02.056

[3] S. Kulkarni, "Nanotechnology—Principles and Processes," Capital Publishing House, New Delhi, 2009, pp. 263-265.

[4] P. Song, Q. Wang and Z. X. Yang, “Ammonia Gas Sensor Based on $\mathrm{PPy} / \mathrm{ZnSnO}_{3}$ Nanocomposites," Materials Letters, Vol. 65, No. 3, 2011, pp. 430-432. doi:10.1016/j.matlet.2010.10.087

[5] N. V. Bhat, M. B. Kurup, V. A. Bambole, M. M. Nate and S. Sabharwal, "Effect of Gamma Irradiation on Structure and Properties of PVA Films," Nuclear Instruments and Methods in Physics Research Section B, Vol. 237, 2005, p. 585. 